\title{
Aux origines de la critique des paperasses
}

\section{Paola Italia}

\section{OpenEdition}

\section{Journals}

Édition électronique

URL : https://journals.openedition.org/genesis/4497

DOI : 10.4000/genesis.4497

ISSN : 2268-1590

\section{Éditeur :}

Presses universitaires de Paris Sorbonne (PUPS), Société internationale de génétique artistique littéraire et scientifique (SIGALES)

\section{Édition imprimée}

Date de publication : 15 décembre 2019

Pagination : 47-59

ISBN : 979-10-231-0650-3

ISSN : 1167-5101

\section{Référence électronique}

Paola Italia, «Aux origines de la critique des paperasses », Genesis [En ligne], 49 | 2019, mis en ligne le 01 décembre 2020, consulté le 12 janvier 2023. URL : http://journals.openedition.org/genesis/4497 ; DOI : https://doi.org/10.4000/genesis.4497 


\section{Aux origines de la critique des paperasses}

Paola Italia

$\mathrm{O}$ n peut aujourd'hui reconstituer la naissance de la critique des variantes - qui fut plus tard nommée «critique des paperasses » par son fondateur Gianfranco Contini (1912-1990) ${ }^{1}$ - et le débat qui suivit son affirmation en Italie à l'aune de documents inédits du fonds Contini et grâce au travail de catalogage et de valorisation de ses papiers et de sa correspondance soutenu par la Fondation Franceschini et par l'École normale supérieure de Pise, sous l'impulsion de Lino Leonardi et de Claudio Ciociola².

Il s'agit d'un moment critique fondamental de l'histoire culturelle italienne. En effet, cette confrontation dialectique avec la vieille génération (celle qui avait résisté au fascisme et dont Benedetto Croce, avec son Manifeste des intellectuels antifascistes de 1925, est le plus célèbre représentant) a dominé la culture italienne jusqu'aux années 1960 - une confrontation parfois âpre, menée par la nouvelle génération des intellectuels italiens, celle de la Résistance, qui tout en admirant Croce ne pouvait pas se reconnaître dans l'idéalisme qu'il représentait.

Gianfranco Contini fut un protagoniste central de ce moment critique. Spécialiste de philologie romane et critique militant, il a uni l'étude philologique du passé à un intérêt pour les poétiques de la modernité, en particulier pour le symbolisme, et a introduit en Italie depuis les années trente une nouvelle idée du texte, entendu comme processus et non plus comme état. C'est justement l'orientation prise par les travaux de l'ITEM - l'institution qui en Europe a le plus œuvré pour garantir la continuité de cette idée -, que pourrait synthétiser la citation placée en exergue de cet article, tirée d'un auteur français qui fut très cher à Contini.

Notre propos est de retracer l'histoire de la naissance de cette idée, en rappelant les liens entre la critique des variantes et la poésie symboliste française, puis les origines communes de la critique des variantes et de la critique génétique, dans le contexte culturel italien de l'après-guerre encore dominé par la personnalité de Benedetto Croce. Ce fut une confrontation très dure entre générations, une véritable révolution culturelle, mais qui, à la différence

1. Sur la naissance de la critique des variantes et son rapport avec la critique génétique, voir M. T. Giaveri, «La critique génétique en Italie : Contini, Croce et l'“étude des paperasses" », Genesis, n 3, 1993, p. 9-29; C. Segre, "Contini, Croce, e la critica degli scartafacci», dans A. Pupino (dir.), Riuscire postcrociani senza essere anticrociani. G. Contini e gli studi letterari del secondo Novecento, Florence, Ed. del Galluzzo, 2004, p. 297-304; M. Ciliberto, Contini, Croce e gli «scartafacci», dans G. Contini, L'influenza culturale di Benedetto Croce, Pise, Ed. della Normale, 2013, p. 571-597. 2. Voir C. Borgia (dir.), Inventario dell'archivio di Gianfranco Contini, Florence, Ed. del Galluzzo, 2012; C. Borgia et F. Zabagli (dir.), Scartafacci di Contini : catalogo della mostra, Florence, Ed. del Galluzzo, 2012; C. Ciociola (dir.), Il giovane Contini, Pise, Ed. della Normale, 2014; L. Leonardi (dir.), Gianfranco Contini 1912-2012. Attualità di un protagonista del Novecento, Florence, Ed. del Galluzzo, 2014. Je remercie Lino Leonardi, directeur de la Fondazione Franceschini, pour m'avoir donné accès aux documents ici présentés, et Riccardo et Roberto Contini pour l'autorisation de les publier; toute ma reconnaissance va également à Claudio Ciociola, attentif lecteur de ces pages. 
d'autres révolutions, ne se montra pas au départ sous un jour violent, hostile à l'autorité ; elle se présenta plutôt comme une autre manière de pratiquer la critique, partant de prémisses différentes pour aboutir aux mêmes résultats. Une sorte de révolution de velours.

C'est l'édition critique des Frammenti autografi dell'Orlando Furioso, procurée par le philologue Santorre Debenedetti en 1937, qui offrit à Contini l'occasion d'affirmer cette nouvelle conception du texte. À vingt-cinq ans seulement, Contini publie le 18 juillet 1937, dans un quotidien peu connu (Il Meridiano di Roma), une recension intitulée «Come lavorava l'Ariosto ${ }^{3}$, qui fonde la critique des variantes. Le titre est emblématique car il reprend celui de deux modèles de la critique proustienne, alors inconnus en Italie ${ }^{4}$ : Comment travaillait Proust, Bibliographie, Variantes, Lettres de Proust de Léon Pierre-Quint (Éditions des Cahiers libres, 1928) et Comment Marcel Proust a composé son roman par Albert Feuillerat (New Haven, Yale University Press, 1934), deux études que Contini utilisera dans ses essais critiques consacrés aux variantes chez Proust ${ }^{5}$.

S'il s'était trouvé en France un lecteur de cet article sur l'Arioste, il l'aurait jugé bien familier. En effet, en ce mois de juillet 1937 au cours duquel Contini avait passé quelques jours à Paris

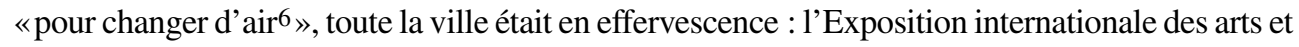
des techniques attirait des visiteurs du monde entier. On remarquait, parmi les pavillons consacrés aux arts, une installation spectaculaire et ambitieuse prenant la forme d'une exposition de manuscrits d'auteurs français modernes dont le titre du catalogue affirmait l'ambition : Ébauche et premiers éléments d'un Musée de la littérature. Les promoteurs en étaient l'administrateur général de la Bibliothèque nationale de France, Julien Cain, et l'académicien Paul Valéry, qui signait la préface du catalogue publié au mois de juillet de l'année suivante7.

Les salles se paraient d'agrandissements géants des manuscrits conservés par la Bibliothèque nationale 8 : on pouvait y admirer les originaux des Fleurs du mal et de la Vie de Henri Brulard, l'autographe de L'Éducation sentimentale et les «paperoles» de la Recherche (fig. 1). Chaque section consacrée à un grand auteur de la littérature française avait été confiée aux soins d'un spécialiste qui présentait, dans le catalogue, l'une des premières analyses génétiques de ses manuscrits 9 . Cette imposante installation fut présentée par ses concepteurs comme

\footnotetext{
3. G. Contini, «Come lavorava l'Ariosto», dans Esercizî di lettura sopra autori contemporanei, con un'appendice su testi non contemporanei, Florence, Parenti, 1939, p. 233-241.

4. M. T. Giaveri (art. cit., p. 15, note 6) relevait la proximité entre l'intervention de Contini et les études proustiennes, tout en observant que ces études pouvaient être «difficilement qualifiées de "proto-génétiques"».

5. G. Contini, «Introduzione alle "paperoles" », dans Letteratura, n’ 36 (nov.-déc. 1947), p. 102-109, puis dans Id., Varianti e altra linguistica, Turin, Einaudi, 1970, p. 60-110.

6. Lettre de Contini à Luigi Russo, 15 juillet 1937, dans D. De Martino (dir.), «Il paesaggio d'un presentista». Corrispondenza tra Gianfranco Contini e Luigi Russo (1936-1961), Florence, Ed. del Galluzzo, 2009, p. 10.

7. Voir la reconstitution minutieuse de Claire Bustarret : «Quand l'écriture vive devient patrimoine : les manuscrits d'écrivains à l'Exposition de 1937 », dans Culture \& Musées, n 16, 2010, p. 159-176. Voir aussi l'étude de Gilles Pitoiset, Les Bibliothèques dans l'Exposition internationale de Paris 1937, Mémoire de fin d'études sous la direction de Noe Richter, École Nationale Supérieure des Bibliothèques, 1983.

8. Le catalogue de l'exposition parut sous le titre Ébauche et premiers éléments d'un Musée de la littérature : Exposition internationale des arts et techniques, Paris, 1937, présenté sous la direction de Julien Cain, Paris, Denoël, 1938. Il est illustré de vingt-quatre photographies de Jean Collas qui restituent la scénographie grandiose de l'installation. 9. Y collaborèrent notamment : Henri Martineau (Stendhal, ibid., p. 3-5), Marcel Bouteron (Balzac, p. 5-13), Cécile Daubray (Hugo, p. 14-19), René Dumesnil (Flaubert, p. 25-31), Maurice Le Blond (Zola, p. 36-38), Léon Pierre-Quint (Proust, p. 50-55)...
} 


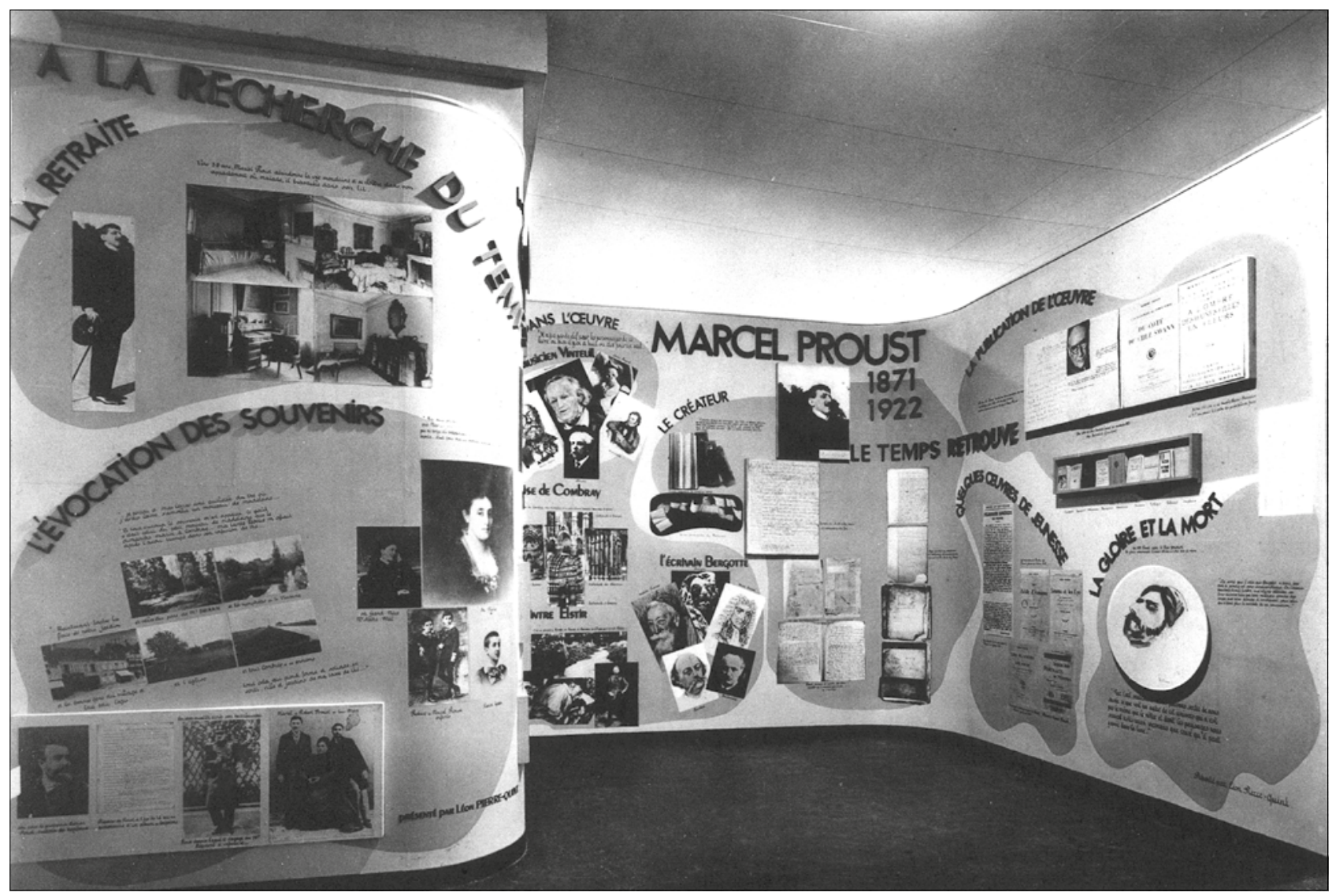

Fig. 1 : Panneaux consacrés à Marcel Proust dans le cadre de l'exposition Ébauche et premiers éléments d'un Musée de la littérature, organisée à la BnF à l'occasion de l'Exposition internationale des arts et des techniques de 1937.

(c) J. Cain (dir.), Ébauche et premiers éléments d'un Musée de la littérature, op. cit. [planches non numérotées]

une nouveauté non seulement littéraire, mais artistique au sens large : le manuscrit, objet jusqu'alors inconnu du grand public, est montré comme le lieu de la création, et permet de mettre en scène une idée nouvelle de la littérature, conçue comme processus, comme acte, dans une perspective expressément pédagogique (comme l'affirmait Julien Cain) que l'on interpréta comme une variante esthétique de la pédagogie démocratique du Front populaire10.

L'introduction du catalogue par Valéry - avec laquelle l'article de Contini «Come lavorava l'Ariosto », rédigé au lendemain de son séjour parisien, présente de fortes résonances thématiques ${ }^{11}$ - nous place directement au cœur d'une nouvelle idée de l'art, où cohabitent fragment et structure, mouvement et monument, et où le manuscrit, au lieu de revêtir les apparences piteuses de l'ébauche et de la paperasse, se trouve magnifié par l'adjonction et l'injonction d'une majuscule : il devient le «Manuscrit original».

10. Sur l'ambition de «rendre accessible à tous une documentation habituellement réservée aux spécialistes et capable de provoquer un plus grand désir de culture», voir C. Bustarret, «Quand l'écriture vive devient patrimoine», art. cit., p. 173 , note 3 .

11. Relevons aussi le singulier jeu d'écho qui unit une note rédigée par Valéry dans ses Cahiers, le 21 janvier 1932, après qu'il eut vu les manuscrits de Hugo à la $\mathrm{BnF}$ («Un poème complet serait le poème de ce Poème à partir de l'embryon fécondé - et les états successifs, les interventions inattendues, les approximations. Voilà la vraie Genèse », éd. Judith Robinson, Paris, Gallimard, Bibliothèque de la Pléiade, t. II, 1974, p. 1118), note que l'on considère comme l'acte de naissance de la critique génétique, et la célèbre définition de la correction comme «approximation de la valeur» dans l'article de Contini de 1937. 
Valéry, avec la conscience de celui qui inaugure non pas une exposition mais une esthétique, présente une «grande et paradoxale nouveauté», capable de «donner à ce principe invisible, à l'esprit même, je ne sais quel visage, et de faire apparaître aux yeux des visiteurs, l'invention elle-même, auprès des choses inventées 12 ». Dans une époque dominée «par la notion de travail mesurable», ces «premiers éléments » d'un Musée de la littérature ont pour mission de «rendre très sensibles l'existence et la dignité du travail qui n'a pas de mesure ${ }^{13}{ }$. Difficile d'imaginer que le Musée de la littérature ait pu échapper au jeune philologue italien qui avait déjà passé à Paris les années 1934-1936 et qui était parvenu, en mars 1936, à entrer en contact avec Valéry grâce à l'intercession d'Emilio Cecchi ${ }^{14}$.

Si Valéry fut par la suite célébré comme le précurseur de la critique génétique 15 et l'Exposition de 1937 comme son acte fondateur, un rôle important échut également à Contini, qui transmit en Italie cette nouvelle dimension artistique, plus encore que critique, dans un contexte historique et culturel certes réceptif à l'étude des variantes ${ }^{16}$, mais pas entièrement disposé (comme nous le verrons) à en reconnaître la nouveauté, ni à s'y montrer favorable. Toute révolutionnaire qu'elle fût, l'initiative de Contini - qui appliquait l'étude des corrections d'auteur au plus prestigieux des poètes de la Renaissance italienne, l'Arioste, fondamental pour la tradition du roman chevaleresque - passa d'abord inaperçue. Ce n'est que dix ans plus tard que ses idées entrèrent dans le cercle du débat critique en Italie, grâce à un long article dans lequel Contini appliquait l'étude des variantes d'auteur à Pétrarque lui-même17. Ce texte s'ouvrait sur une prise de position provocatrice, qui plaçait l'étude des variantes dans un contexte théorique plus général et redéfinissait l'idée même du texte18 :

L'école poétique issue de Mallarmé, et qui trouve en Valéry son théoricien, parce qu'elle considère la création poétique dans son devenir, l'interprète comme un travail perpétuellement en mouvement, sans achèvement, dont le poème historique représente une section possible, arbitraire, et pas nécessairement définitive. C'est un point de vue de producteur, non de destinataire. Si toutefois le critique envisage l'œuvre d'art comme un «objet», cela ne représente que l'objectivité de son approche, le «donné» est l'hypothèse de travail morale de son abnégation; et la prise en considération de l'acte poétique le conduira à déplacer dynamiquement ses formules, à repérer les orientations plutôt que les contours fixes de l'énergie poétique ${ }^{19}$.

12. P. Valéry, Préface, dans Ébauche et premiers éléments d'un Musée de la littérature, op. cit., p. VI.

13. Ibid., p. V-VI.

14. Voir le récit de leur entrevue, en date du 6 juin 1936, dans P. Leoncini (dir.), L'onestà sperimentale : carteggio di Emilio Cecchi e Gianfranco Contini, Milan, Adelphi, 2000, p. 31-32.

15. Voir J. Robinson-Valéry, «Valéry précurseur de la génétique», Genesis, n 5, 1994, p. 89-98.

16. Voir C. Ciociola, «Storia della tradizione e varianti d'autore (Barbi, Pasquali, Contini)», dans C. Ciociola, C. Vela (dir.), La tradizione dei testi, Florence, Società dei Filologi della Letteratura Italiana, 2018, p. 3-22.

17. G. Contini, Saggio d'un commento alle correzioni del Petrarca volgare (1941), Florence, Sansoni, 1943.

18. Rappelons les considérations de Louis Hay sur le lien entre la pratique de la correction chez Pétrarque et les modalités créatives présentes dans les manuscrits modernes : «Dans les manuscrits de Pétrarque apparaît une pratique d'écriture débridée qui évoque déjà celle de certaines pages contemporaines. La porte est ainsi ouverte à la forme la plus complexe de l'écriture : le brouillon de genèse» (L. Hay, «Défense et illustration de la page », Genesis, $\mathrm{n}^{\circ} 37,2013$, p. 33-53).

19. G. Contini, «Saggio d'un commento alle correzioni del Petrarca volgare», dans Varianti e altra linguistica, op. cit., p. 5 (nous traduisons). 
Le langage de Contini est énigmatique et nécessite une sorte de «traduction». L'écrivain Carlo Emilio Gadda, maître du plurilinguisme, après avoir lu la recension de l'une de ses œuvres par Contini, écrivit à sa sœur qu'il fallait lire le texte «quatre fois avant d'y comprendre quelque chose 20 »... Essayons de traduire. La poésie est un processus. Le texte sur lequel nous exerçons notre activité critique, le «donné», donc, est une «section», un fragment, un moment de ce processus, qui peut ne pas coïncider avec la dernière volonté de l'auteur. La valeur d'un texte ne réside pas dans son état final, mais dans son devenir œuvre. Ce que le critique a toujours considéré comme un «objet», ce n'est pas le «poème historique», c'est-à-dire la manifestation historique du texte, mais l'objet mental sur lequel s'applique son activité critique («l'hypothèse de travail morale de son abnégation»). L'objectivité est donc dans l'activité mentale, et non dans l'historicité du «donné», qui est, au contraire, un flux, un devenir, de sorte que l'activité critique doit être dédiée à identifier les «orientations » plutôt que les «contours fixes de l'énergie poétique». Comme on peut le voir, il s'agit d'une conception qui doit beaucoup aux poétiques symbolistes de Mallarmé et de Valéry, expressément citées.

La guerre retarde la diffusion de ces idées mais n'en réduit pas l'importance; on pourrait même dire qu'elle leur donne une dimension «européenne ${ }^{21}$ ». Contini, qui occupe à partir de 1938 la chaire de linguistique italienne à Fribourg et qui prend part en 1944 à la Résistance dans la République de l'Ossola, tient en Suisse une série de conférences dont ne nous sont parvenues que des notes. L'étude de ces brouillons, déposés aujourd'hui aux Archives Contini - et qui constitue en somme une «critique des variantes» de la «critique des variantes», vertigineuse mise en abyme - nous permet de mieux comprendre la signification théorique des essais de Contini sur l'Arioste et sur Pétrarque. L'une de ces pages manuscrites - présentée en avant-première à Paris en 2015, à l'occasion du colloque de l'ITEM sur les Manuscrits italiens du XVIII e siècle 22 - concerne justement la Portée théorique de l'étude des corrections. Parcourons brièvement les concepts exposés dans ce feuillet manuscrit :

Portée théorique de l'étude des corrections

$[\ldots]$

Portée philosophique :

ou la poésie est un être, une présence absolue (texte $=$ objet)

ou elle est un devoir être, une évolution infinie (Mallarmé, mais surtout Valéry)

l'étude des corrections correspond au $2^{\mathrm{e}}$ aspect, mais ses résultats doivent concorder avec l'étude du 1 er.

il ne s'agit pas seulement de marquer le hiatus, la discontinuité du génie mais d'identifier l'orientation générale et unitaire, et surtout le renoncement aux fragments valides pour la totalité valide 23

20. P. Italia, «Quarta giunta al carteggio Contini-Gadda, ovvero di Contini 4, 5, 6», dans L. Leonardi (dir.), Gianfranco Contini 1912-2012. Attualità di un protagonista del Novecento, Florence, Ed. del Galluzzo, p. 144-176.

21. Voir G. Contini, Dove va la cultura europea? Relazione sulle cose di Ginevra, éd. L. Baranelli, Macerata, Quodlibet, 2012.

22. Voir P. Italia, «Filologia d'autore, critica genetica e critica delle varianti : diacronia, sincronia e tassonomia», dans C. Del Vento et N. Ferrand (dir.), I manoscritti italiani del XVIII secolo : un approccio genetico, Florence, Le Lettere, p. 33-48.

23. Florence, Fondazione Ezio Franceschini, Fonds Contini, série 7, fascicule 44 (II.76) (nous traduisons). 
Dans l'après-guerre, cette nouvelle théorie qui opposait le texte perçu comme «processus » ou «évolution infinie» au texte entendu comme «objet» aux «contours fixes» est interprétée comme une atteinte à l'idéalisme de Croce. Elle apparaît comme le fer de lance d'une «nouvelle critique» qui risquait de mettre à mal la tradition italienne, en donnant à cette tradition un pedigree étranger.

Il est vrai que Contini tire cette idée du texte comme «devoir-être, évolution infinie ${ }^{24}$ » de Mallarmé et Valéry, en se référant à la tradition du symbolisme français, mais il va aussi jusqu'à appliquer cette nouvelle méthode critique aux auteurs moralement réprouvés et pour ainsi dire mis à l'index par l'orthodoxie de Croce : Mallarmé toujours (que Croce considérait comme un «maniaque 25 ») et Proust (liquidé par Croce comme un imitateur de Maupassant).

Il suffit de parcourir le sommaire de la section «Critique des variantes» du recueil Varianti e altra linguistica, publié par Einaudi en 1970, pour se rendre compte à quel point cette prise de position fut courageuse et provocatrice. Après les trois premiers essais, consacrés à Pétrarque, Manzoni et Leopardi, suivent trois essais consacrés à Mallarmé et à Proust :

Critique des variantes

Essai de commentaire des corrections de Pétrarque en «langue vulgaire»

Corrections grammaticales chez Pétrarque

Un livre d'étrennes de Manzoni

Implications léopardiennes

Sur la transformation de L'Après-midi d'un faune

Introduction aux «paperoles»

Jean Santeuil, ou l'enfance de la Recherche

Cette provocation suscite une double polémique : en 1947, Benedetto Croce publie un article virulent, intitulé «Illusions sur la genèse des œuvres d'art, documentée par les paperasses des écrivains ${ }^{26}$ », qui manifeste son dédain envers la jeune critique des variantes, considérée comme un passe-temps amusant bien éloigné de la critique littéraire; puis en 1948, Nullo Minissi, disciple de Croce, fait paraître une violente attaque personnelle contre Contini ( Les corrections et la critique 27 »), qui lui répond dans son essai La Critica degli scartafacci [La Critique des paperasses] dont le titre reprend à dessein le terme «scartafacci » utilisé par Croce 28 .

Acte fondateur de cette nouvelle méthode critique, l'essai La Critique des paperasses ne fut pourtant jamais publié en volume, subissant une forme de rejet de la part de Contini lui-même ${ }^{29}$. Ce dernier ne se présentait d'ailleurs pas comme le fondateur de la nouvelle critique, préférant, dans l'article «Philologie » qu'il composa vingt-cinq ans plus tard pour l'édition révisée de l'Encyclopédie italienne, rappeler les précédents français et allemands.

24. G. Contini, Varianti e altra linguistica, op. cit., p. 5.

25. B. Croce, recension de Mallarmé de Paul Valéry, dans Quaderni della Critica, a. III, n 9, 1947, p. 77-79.

26. Id., «Illusione sulla genesi delle opere d'arte, documentata dagli scartafacci degli scrittori », dans Quaderni della Critica, a. III, n 9, 1947, p. 93-94.

27. N. Minissi, «Le correzioni e la critica», dans Belfagor, III, 1948, p. 94-97.

28. G. Contini, «La critica degli scartafacci», dans Rassegna d'Italia, a. III, n 10 et 11 (oct. et nov. 1948), p. $1048-1056$ et p. $1156-1160$.

29. Le texte ne sera réédité qu'en 1992 : G. Contini, La Critica degli scartafacci e altre pagine sparse, Pise, Scuola Normale Superiore, 1992. 


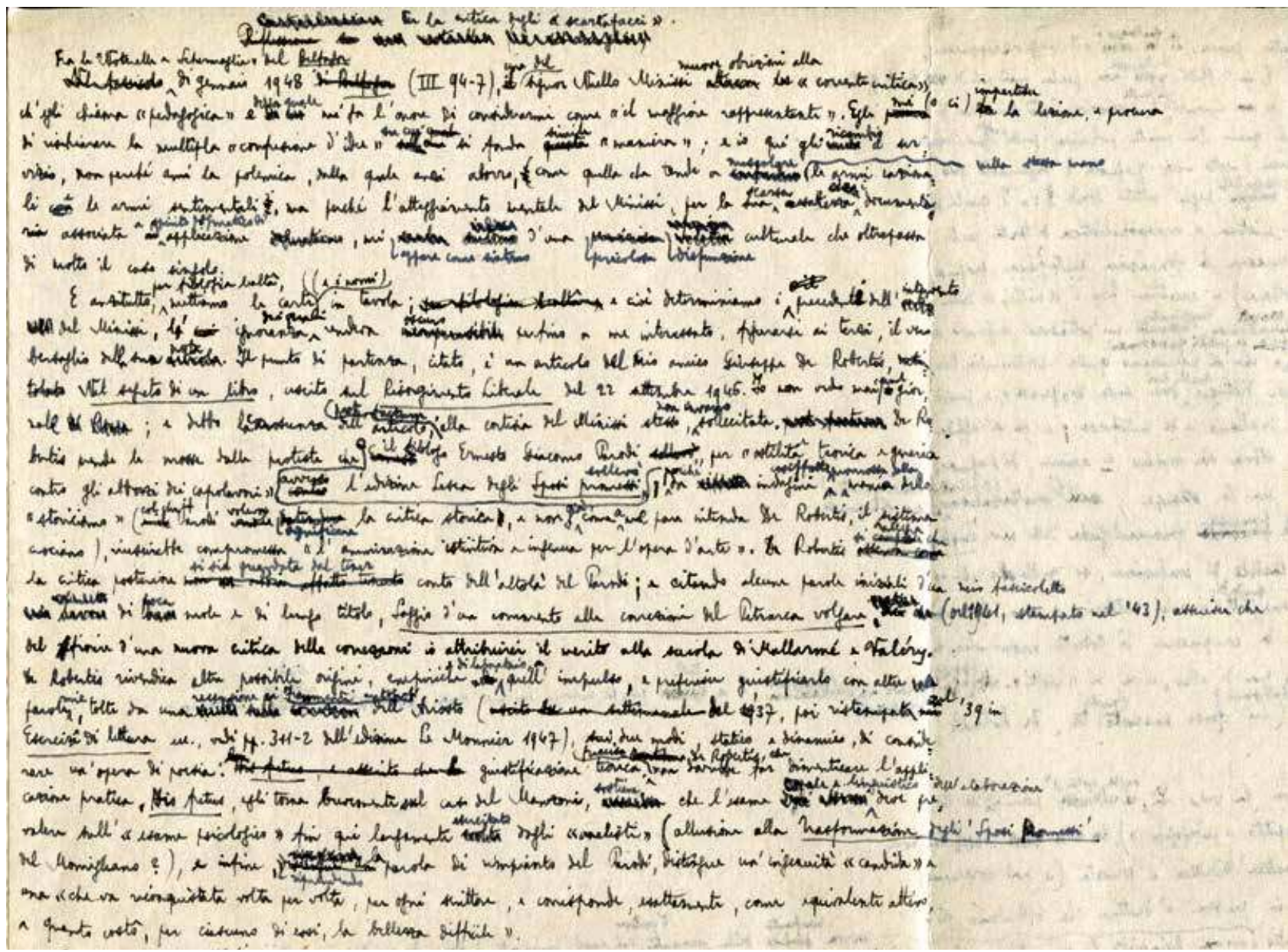

Fig. 2 : «Per la critica degli "scartafacci”» [« Pour la critique des "paperasses”»], manuscrit f. $1 \mathrm{r}^{\circ}$. Florence, Fondazione Ezio Franceschini, Fonds Contini (détail); voir C. Borgia, op. cit., $\mathrm{n}^{\circ} 45$, p. 37. Le titre «Riflessioni su una notarella (in margine)» [«Réflexions sur une petite note (en marge)»], effacé par 1'auteur, est remplacé par «La Critica degli "scartafacci” » [ La Critique des "paperasses"»].

Le modèle français était à ses yeux le «petit traité» d'Antoine Albalat, Le travail du style enseigné par les corrections manuscrites des grands écrivains (première édition de 1903), qui applique des analyses «souvent passionnantes» aux variantes de Chateaubriand et de Victor Hugo. Le modèle allemand se déclinait à travers trois critiques : Hanns Heiss, qui applique une approche idéaliste au même Victor Hugo; Arthur Franz, qui théorise une «typologie dynamique

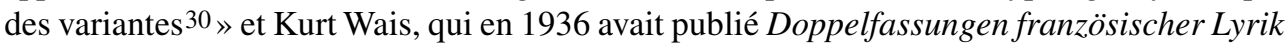
von Marot bis Valéry [Doubles rédactions de poésie française de Marot à Valéry].

Le manuscrit de La Critique des paperasses consiste en neuf feuillets couverts d'une écriture serrée, qui présentent une double série de corrections : la première, qui suit de peu la rédaction, est effectuée avec le même stylo, la seconde est plus tardive, l'encre du stylo est plus claire ${ }^{31}$ (fig. 2). La version finale, qui correspond à la transcription dactylographiée

Le manuscrit de La Critique des paperasses

30. G. Contini, Varianti e altra linguistica, op. cit., p. 18 (nous traduisons).

31. C. Borgia, Inventario dell'archivio di Granfranco Contini, op. cit., ${ }^{\circ}$ 45, p. 37 (Archivio Contini, II, 75, Bibliografia 45, f. 1-9). 
effectuée par Contini lui-même 32 (fig. 3), n'est pas très différente de celle qui fut publiée dans la «Rassegna d'Italia» d'octobre-novembre 1948. La comparaison entre le premier état manuscrit de La Critique des paperasses et sa version finale est extrêmement utile pour mettre en évidence les véritables intentions du texte. Les axes de l'argumentation de Contini sont au nombre de trois :

- le premier concerne la tentative de minorer son propre rôle de fondateur de la critique des variantes, qualifiée par Nullo Minissi de critique «pédagogique»;

- le deuxième retrace la genèse de cette critique, en évitant de la ramener uniquement à la ligne Mallarmé-Valéry;

- le troisième soutient que la critique des variantes n'est pas contraire à la critique de Croce : elle est différente par la méthode, mais identique par ses résultats.

D'un côté, Contini semble donc vouloir se mettre à l'écart, en refusant d'être considéré comme le fondateur de la critique des variantes et en partageant avec d'autres la responsabilité de la «méthode»; mais d'un autre côté - et l'analyse de ses corrections le montre - il ne parvient pas à éviter l'effet inverse. Cesare Segre, qui, avec Dante Isella, a mené la réflexion la plus aboutie sur la critique des variantes et la philologie d'auteur, a soutenu en 2004 que l'on pouvait discerner une subtile autocensure derrière la polémique ${ }^{33}$, tandis qu'Isella a parlé de «simple différenciation empirique » entre les deux méthodes critiques ${ }^{34}$. L'évolution du titre corrobore ce double constat. Il se présentait au départ sous une forme neutre et métatextuelle : Réflexions sur une petite note (en marge) (fig. 2). Puis il devient, par le biais de la reprise ironique du terme polémique de Croce «scartafacci», la magna charta de la nouvelle critique : La Critica degli «scartafacci» [La Critique des «paperasses»].

Suivons de près l'argumentation. Après avoir déclaré qu'il ne voulait pas jouer le rôle de «porte-parole» de la nouvelle méthode, Contini cherche à réduire l'importance de la dérivation de Mallarmé-Valéry. Mais il lui était difficile de ne pas manifester son estime pour celui qu'il considérait comme un maître. Ainsi, s'il minimise dans son essai sur les brouillons ce qu'il doit à la critique des variantes issue de la poésie symboliste française, d'un autre côté, il manifeste sa francophilie en participant au numéro conclusif de la revue Letteratura de novembre-décembre 1947 dédié à Proust, avec une exigeante «Introduction aux "paperoles" », rigoureusement fidèle à la méthode déjà expérimentée pour Pétrarque, l'Arioste, Manzoni et Leopardi. C'est un camouflet infligé au camp de Croce, porté sur le terrain même du roman, et dont Contini sait à quel point il peut être dangereux. Il suffit de suivre la relation presque médicale qu'il en fait dans le post-scriptum d'une vibrante lettre à Cecchi, écrite le 12 octobre 1947 :

Je devais terminer un papier pour le numéro proustien de Letteratura, un papier dont j'avais dû plusieurs fois interrompre la rédaction par nécessité impérieuse; et je sentais avec une terreur

32. Les Archives Contini conservent une première transcription dactylographiée incomplète, à l'encre rouge (trois feuilles seulement), et une seconde transcription dactylographiée complète de onze feuilles portant des corrections manuscrites sporadiques.

33. C. Segre, «Contini, Croce, e la critica degli scartafacci», art. cit., p. 303.

34. D. Isella, «Le varianti d'autore (critica e filologia)» (1984), dans Id., Le carte mescolate vecchie e nuove, éd. S. Isella Brusamolino, Turin, Einaudi, 2009, p. 13 (nous traduisons). Voir aussi, du même auteur «Ancora sulla critica delle varianti » (1990), dans Id., L’idillio di Meulan. Da Manzoni a Sereni, Turin, Einaudi 1994, p. 306-323. 
Per la critica degli "Bcartafacci"

Bra le "roterelle e Schermaglie" del Belfagor di gennaio 1848 (III 94-7), una del signor Nullo Minissi move obiezioni alla "corrente critica" ch'egli chiama "pedagogica" e della quale mi fa l'onore di considerami come il "mag giore rappresentante". Bgli mi (o ci) impartisce la lezione, e procura di rischiarare la multipla "confusione d'idee" su cui si fonda simile "maniera"; io qui gli ricambio 11 servizio, non perché ami la polemica, dalla quale anzi aborro, come quella che tende a mescolare nella stessa mano le armi razionall e le sentimentali, ma perché l'atteggiamento mentale del uinissi, per la scarsa esattezza circa 1 documenti associata a spirito dogmatico di applicazione, mi appare come sintomo d'una pericolosa disfunzione culturale che oltrepassa di molto il caso singolo.

$\mathrm{E}$ anzitutto, per filologica lealtà, mettiamo le carte (e 1 nomi) in tavola; e cioé determiniamo $i$ precedenti dell'intervento del linissi, l'ignoranza dei quali rendeva oscuro perfino a me interessato, figurarsi ai terzi, 11 vero ber saglio della sua nota. Il punto di partenza, citato, é un articolo del mio ami. co Giuseppe DeRobertis, Nel segreto di un libro, uscito sul Risorgimento liberale del 22 settembre 1946 . Io non vedo mai qual giornale; e debbo la conoscen za dell'articolo, post factum, alla cortesia del Minissi, non invano sollecite ta. DeRobertis prende le mosse dalle proteste che avverso l'edizione lesca degli Sposi promessi a ollevò il filologo Brnesto Giacomo Parodi, per "ostilità teorica e generica contro gli abbozzi del capolavori", poiché da indagini cosiffatte, promosse dalla mania dello "storicismo" (col che il Parodi voleva significare la critica storica, e non già, come a me pare intenda DeRobertis, 11 gistema crociano), riuscirebbe compromessa "l'aimirazione istintiva e ingenua per l'opera d'arte". DeRobertis si rallegra che la critica posteriore si sia guardeta dal tener conto dell'altolà del Parodi; e citando alcune parole iniziali d'un mio fascicoletto di poca nole e di lungo titolo, Saggio d'un comento alle correzioni del Petrarca volgare (del 1941, stampato nel 143), asserisce che del fiorire d'una nuova critica delle correzioni io assegnerei il merito alla scuola di Nallarmé e Valéry. DeRobertia rivendica altre possibi ii origini, empiriche e di laboratorio, a quell'impulso, e preferisce giustifi carîo con altre parole mie, tolte da una recensione ai Frammenti autografi del I'Ariosto (del '37, poi ristampata nel ' 39 in Bsercizi di lettura ecc.,vedi pp.311-2 delledizione Lelionnier 1947), sui due modi, statico e dinamico, di considerare un'opera di poesia, giustificazione teorioa, preaisa DeRobertis, che non dovrebbe far dimenticare l'applicazione pratica. His fretus, egli torna brevemente sul caso del llanzoni, sostiene che l'esame tonale e linguistico dell'elaborazione deve prevalere sull"esame psicologico" fin qui largamente esercitato dagli "analisti" (allusione alla Trasformazione degli 'Sposi promes si' del Komigliano ?), e infine, riprendendo la parola di rimpianto sfugsita al parodi, distingue un'ingenuità "candida" e una "che va riconquistata volta per volta, per ogni scrittore, e corrisponde, esattanente, come equivalente attivo, a quanto eostò, per ciascuno di essi,la bellezza difficile".

Prima di fornire occasione alla nota del linissi, il pezzo di DeRobertis aveva itritato Benedetto Croce, la cui replica (intitolata Illusione sulla genesi delle opere d'arte, documentata dagli scartafacci degli scrittori, e chie ramente allusiva \& quell'articolo pur senza citarlo in tutte le lettere) puo leggersi nel $9^{\circ}$ dei Quadermi della Critica (1947), pp.93-4. Il Croce ramenta d'aver diviso le preoccupazioni del Parodi, prende atto che lo studio delle correzioni é ora passato nelle mani dei "decadenti" amiratori di liallarmé e Valery, nega che la genesi documentaria sia genetica perché il pri-

Fig. 3. «Per la critica degli "scartafacci"» [« Pour la critique des "paperasses"»], tapuscrit. Florence, Fondazione Ezio Franceschini, Fonds Contini; voir C. Borgia, op. cit., n 45, p. 37. 
superstitieuse, alors que ma nervosité allait croissant, que si je m'en éloignais encore une fois, ne fût-ce que pour écrire une seule lettre, si je ne liquidais pas l'hypothèque le plus rapidement possible, il n'y aurait plus rien à faire. Ainsi, une fois apposé l'ultime signe de ponctuation, j'ai expédié le produit informe sans même le nettoyer : comme l'œuf qui arrive du poulailler, encore sale de sang et d'excréments. Tu tiendras compte de cette préhistoire pour le juger 35 .

Nous devrons, nous aussi, tenir compte de cette image qui renvoie à un amalgame matériel, corporel, entre l'auteur, le texte et le critique. Dans son essai proustien, Contini s'était confronté au monde littéraire avec beaucoup d'effronterie : il avait dû rappeler, face au reproche d' «indiscrétion posthume» brandi contre la critique des variantes accusée de fureter sans vergogne ni respect dans les manuscrits d'auteurs, le soin zélé avec lequel Proust avait conservé ses papiers; et il avait même soutenu que l'étude de ces «paperoles» permettait d'éviter deux erreurs d'interprétation : «prendre la Recherche pour un livre de souvenirs (au lieu de la considérer comme une théorie en acte de la mémoire) et y découvrir une vision minutieuse et macroscopique de la réalité (au lieu d'une vision télescopique et de ce fait même législative) $)^{36}$ ».

$\mathrm{Si}$, d'un côté, ses modèles critiques - Comment travaillait Proust de Quint et Comment Marcel Proust a composé son roman de Feuillerat - disparaissent de son essai sur les paperasses, il suit toutefois dans son analyse des variantes proustiennes de 1947 la méthode qu'il avait appliquée à l'Arioste dix ans plus tôt, comme s'il voulait revendiquer sa préséance, au moment même où il la désavoue.

Dans la partie finale de son texte, Contini revendique l'idée d'une convergence avec la critique de Croce. L'aspect central de son argumentation est relatif à la distinction entre variantes instauratives et variantes substitutives : une double catégorie interprétée de bien des façons, mais dont l'analyse du manuscrit éclaire le sens. Minissi avait en effet soutenu que les véritables variantes sont les variantes instauratives, qui vont du non-être à l'être poétique, tandis que les secondes - qui substituent à des éléments provisoires et «fragmentairement valides » d'autres éléments définitifs et «organiquement valides»-doivent être considérées comme «d'autres textes» du même auteur, des textes véritables, chacun doté de sa spécificité. Minissi, qui n'a aucune expérience en philologie, ne comprend pas ce que Contini avait écrit. Et il ne comprend pas davantage son maître. Pour Croce, en effet, les variantes instauratives ne sont rien d'autre que le résidu incompréhensible d'un processus mental propre à l'auteur, impossible à reconstituer, un processus dont ne restent que des bribes déconnectées, qui ne peuvent révéler l'essence de sa poésie. On ne peut donc formuler aucun jugement esthétique sur ces bribes, car, dans le parcours qui conduit «du non-être à l'être poétique», il n’y a pas de poésie.

Il n'en va pas de même pour les variantes substitutives, que privilégie Contini. Dans son analyse des manuscrits modernes, de Mallarmé à Proust, la comparaison s'établit principalement entre la parution en revue et le livre imprimé. Rien de plus «substitutif». Cela n'exclut pas, répète Contini, qu'il soit également possible d'identifier des fragments poétiques dans les variantes instauratives : après avoir fourbi ses armes sur Pétrarque (mais avec des comparaisons significatives de textes «achevés»), il cite à titre d'exemple - comme s'il

35. P. Leoncini (dir.), L'onestà sperimentale, op. cit., p. 77 (nous traduisons).

36. G. Contini, Varianti e altra linguistica, op. cit., p. 73 (nous traduisons). 
dénonçait ses complices - les variantes d'Ungaretti étudiées par Domenico De Robertis, celles du Tasse par Lanfranco Caretti, en rappelant que «la dernière volonté de l'auteur » est mise en crise par la perte de la «dimension téléologique» de l'œuvre d'art, et que chaque fragment de texte est en lui-même potentiellement porteur de poésie, puisqu'il représente une approximation de la valeur poétique. Ainsi, l'étude des corrections instauratives pourrait déboucher sur une description caractérisante, qui, loin de s'opposer à la critique esthétique, l'enrichit de données positives et vérifiables.

Contini réaffirme, dans la partie finale de son essai, n'avoir pas voulu fonder une nouvelle esthétique, ni même «proposer un nouveau canon méthodologique »; il rappelle n'avoir jamais prétendu, dans son essai Come lavorava l'Ariosto, «qu'il s'agissait d'une méthode nouvelle, mais bien d'un aspect de la même méthode [celle de Croce] prise dans un sens pédagogique ». Il est significatif que ces dernières phrases aient été ajoutées à la version définitive du manuscrit, comme pour clarifier la question et désamorcer la polémique. Il ne s'agissait pas, poursuit-il, de donner naissance à une «hérésie nouvelle », car une telle nouveauté n'avait pas même été relevée par Croce à l'époque de la recension sur l'Arioste, comme le prouve l'anecdote relatée par Contini à sa décharge (ici en caractères gras les variantes entre la version manuscrite, à gauche, et la version publiée, à droite) :

S'il m'est permis de citer une anecdote, je rappellerai que Croce, quand je fis mon compte rendu des Frammenti autografi de l'Arioste, le chef d'œuvre philologique de Santorre Debenedetti, me $<\ldots>$ qu'il ne ferait pas, pour sa part, de compte rendu de ce livre car j'en avais extrait tout le suc, comme il me le dit avec trop de bienveillance : signe, oserais-je dire, qu'il n'y avait pas rencontré de profonds défauts de pensée.

Quelqu'un d'autre, en revanche, un soir où je parlai de la genèse des Promessi Sposi, me reprocha cette modestie excessive, ce renoncement à bâtir une nouvelle esthétique, au point presque de me reprocher d'être réformiste et non révolutionnaire.
S'il m'est permis de citer une anecdote personnelle, je rappellerai que Croce lui-même, quand j'eus publié cette glose sommaire du chef-d'œuvre philologique de mon maître Santorre Debenedetti, me dit qu'il ne ferait pas, pour sa part, de compte rendu de ce livre car j'en avais extrait tout le suc, selon son expression trop bienveillante : signe, oserais-je inférer, qu'il ne lui avait pas semblé rencontrer de graves anomalies de pensée.

Quelqu'un d'autre, en revanche, un soir où j'eus à parler de l'élaboration des Promessi Sposi, taxa de modestie excessive ce renoncement à bâtir une nouvelle esthétique, au point presque de me reprocher d'être réformiste et non révolutionnaire.

Il n'est pas difficile de reconnaître dans ces conversations le salon littéraire florentin de De Robertis, que Contini mentionne plusieurs fois dans ses lettres à Cecchi, et où on lui aurait reproché sa défense trop timide de la méthode historico-philologique contre l'école critico-idéaliste.

Pourquoi alors - pourrait-on se demander - toute cette polémique, si Contini sait que sa position n'est pas opposée à celle de Croce, dont elle ne constitue qu'une variante «pédagogique »? La réponse est contenue justement dans l'adjectif «pédagogique»: une critique de ce type, capable de conjuguer les approches stylistique et psychologique, la donnée matérielle et son interprétation, était en mesure d'attirer les jeunes et de constituer, pour les nouvelles recrues de la critique, ce pôle d'attraction que ni Croce ni l'idéalisme crocien n'étaient en mesure d'incarner, à présent qu'ils s'étaient appauvris dans un vague téléologisme. Attirer les nouvelles générations, mais avec des modèles culturels décadents et contestés.

C'est ainsi que Proust (qui avait donné lieu à un essai en cours de révision au moment même où Contini écrivait sa réponse à Minissi), après être sorti par la porte, revenait par 
la fenêtre en compagnie de Valéry et de Mallarmé, minorés en apparence, mais considérés comme les initiateurs de la méthode de la «critique des corrections». Une méthode qui allait produire un changement radical dans les études littéraires (et universitaires), en substituant aux monographies classiques - «contenants» insatisfaisants et exposés au risque d'une interprétation psychologique - des analyses linguistiques et stylistiques : des «exercices de lecture » à comprendre comme des formes de critique non moins valables que celle qui examinait, dans les monographies traditionnelles, la «personnalité empirique des auteurs ».

Traduit de l'italien par Claire Riffard, avec la collaboration de Pierre Musitelli

PaOla Italia enseigne la littérature italienne à l'université de Bologne. Ses travaux portent sur la philologie d'auteur (www. filologiadautore.it), dont elle a exploré les enjeux méthodologiques (Che cos'è la filologia d'autore, avec Giulia Raboni, 2010) et qu'elle a appliqué à l'étude des archives littéraires italiennes (XVIII-XXe siècles). Elle a collaboré aux éditions critique de Fermo et Lucia de Manzoni et des Canti de Leopardi, et elle a consacré ses recherches à de nombreux auteurs du xxe siècle (Gadda, Savinio, Manganelli, Sinigaglia, Bassani, Tobino...), dont elle a édité plusieurs ouvrages, et dont elle a étudié la méthode d'écriture (parmi ses derniers travaux: Come lavorava Gadda, 2017).

paola.italia@unibo.it 


\section{Aux origines de la critique des paperasses}

La critique génétique en France et la philologie d'auteur en Italie trouvent dans la critique des variantes de Gianfranco Contini surnommée «critique des paperasses» à l'occasion d'une polémique avec Benedetto Croce - une origine commune. Elle remonte, d'une part, à l'étude des corrections d'auteurs que Contini a appliquée à des écrivains aussi bien italiens (l'Arioste, 1937; Pétrarque, 1941; Leopardi, 1947) que français (Proust, 1947; Mallarmé, 1948). Elle se forme, d'autre part, dans le sillage de ses réflexions sur la « signification théorique de l'étude des corrections », étude qui s'intéresse au « devoir être » du texte et à l' «évolution infinie» de la poésie, en référence à Mallarmé mais surtout à Valéry, comme l'a montré le manuscrit d'une conférence que Contini prononça en Suisse en 1944. La présente contribution retrace l'origine de ces idées à travers l'étude des corrections du manuscrit de La critica degli scartafacci ( «La critique des paperasses», article publié par Contini en 1948), tout en offrant un exemple éclairant - une véritable mise en abyme - de la façon dont travaillait Contini.

The French critique génétique and the Italian filologia d'autore share a common origin: the "variant-based criticism" of Gianfranco Contini - that he also named, on the occasion of a polemic with Croce, the "foul papers criticism" - which could be traced back on the one hand, to the study of authors' corrections that he applied to Italian writers, such as Ariosto (1937), Petrarca (1941) and Leopardi (1947), as well as to French writers, such as Proust (1947) and Mallarmé (1948); and, on the other hand, to his reflections on the "theoretical meaning of the study of corrections", whose philosophical impact lays on the "must be" and on the "infinite evolution" of poetry, with reference to Mallarmé and especially to Valéry, as demonstrated by the manuscript of a lecture he gave in Switzerland in 1944. This paper retraces the birth of those ideas by analysing the corrections to the manuscript of the "foul papers criticism" (the article published by Contini in 1948, in conflict with Croce), also offering a perfect example -a real mise en abyme - of "how Contini worked".

Die französische „critique génétique“ und die italienische Autorenphilologie finden in den „,critique des variantes“ von Gianfranco Contini - anlässlich einer Kontroverse mit Benedetto Croce auch „Kritik der Entwürfe“ genannt - einen gemeinsamen Ursprung. Einerseits geht sie auf die Untersuchung der Korrekturen von Autoren zurück, die Contini sowohl bei italienischen Schriftstellern (Ariosto 1937, Petrarca 1941, Leopardi 1947) als auch bei französischen Schriftstellern (Proust 1947, Mallarmé 1948 ) betrieb. Andererseits entsteht sie in Folge seiner Überlegungen zur ,theoretischen Bedeutung des Studiums der Korrekturen“, einer Studie, die sich auf das „Sein Müssen“ des Textes und die „unendliche Entwicklung“ der Poesie konzentriert. Wie anhand des Manuskripts eines Vortrags, den er im Jahr 1944 in der Schweiz hielt, gezeigt werden kann, nimmt Contini Bezug auf Mallarmé und vor allem auf Valéry. Der vorliegende Beitrag zeichnet die Entstehung dieser Ideen anhand der Korrekturen am Manuskript über die „Kritik der Entwürfe“ (,La critica degli scartafacci“, Artikel von Contini aus dem Jahr 1948) nach und gibt gleichzeitig ein anschauliches Beispiel - eine reale mise en abyme - für die Arbeitsweise von Contini.
La crítica genética en Francia y la filología de autor en Italia encuentran en la crítica de las variantes de Gianfranco Contini -apodada "crítica de los papeleríos" con ocasión de una polémica con Benedetto Croce- un origen común. Éste parte, por un lado, del estudio de las correcciones de autores que Contini dedicó a escritores tanto italianos (Ariosto, 1937; Petrarca, 1941; Leopardi, 1947), como franceses (Proust, 1947; Mallarmé, 1948), y se conforma, por otro lado, en la estela de sus reflexiones sobre la "significación teórica del estudio de las correcciones", reflexiones que se centran en el "deber ser" del texto y en la "evolución infinita" de la poesía, refiriéndose a Mallarmé, y sobre todo a Valéry, como lo pone de manifiesto el manuscrito de una conferencia que Contini dio en Suiza en 1944. El presente trabajo rastrea el origen de estas ideas a través del estudio de las correcciones del manuscrito de La critica degli scartafacci ("La crítica de los papeleríos", artículo publicado por Contini en 1948), brindando un ejemplo revelador -una verdadera mise en abyme- del método de trabajo de Contini.

A crítica genética na França e a filologia do autor na Itália encontram na crítica das variantes, de Gianfranco Contini - o que ele também nomeou «crítica das papeladas» diante de uma polêmica com Benedetto Croce - uma origem comum. Esta, por um lado, pode ser localizada no estudo das correções de autores, que Contini aplicou tanto aos escritores italianos (Ariosto, 1937; Petrarca, 1941; Leopardi, 1947), como aos escritores franceses (Proust, 1947; Mallarmé, 1948), e também, nos vestígios de suas reflexões sobre «o significado teórico do estudo das correções », onde reconhece a sua dimensão filosófica no «dever ser» do texto e na «evolução infinita» da poesia, fazendo referência a Mallarmé, e principalmente a Valéry, como demonstrado no manuscrito de uma conferência que Contini pronunciou na Suiça, em 1944. A presente contribuição retraça a origem dessas ideias através do estudo das correções do manuscrito de La critica degli scartafacci ( «A crítica das papeladas», artigo publicado por Contini, em 1948, em desacordo com Croce) apresentando também, um exemplo esclarecedor - uma verdadeira mise en abyme da «maneira como Contini trabalhava».

La critique génétique francese e la filologia d'autore italiana hanno nella critica delle varianti di Gianfranco Contini - da lui, in polemica con Benedetto Croce, denominata "critica degli scartafacci" - una matrice storica comune, che va fatta risalire allo studio delle correzioni d'autore applicato a scrittori tanto italiani, come Ariosto (1937), Petrarca (1941) e Leopardi (1947), che francesi, come Proust (1947) e Mallarmé (1948), ma anche alle riflessioni sul "significato teorico dello studio delle correzioni", che ne riconosce la portata filosofica nel "dover essere", nella "evoluzione infinita" della poesia, risalente a Mallarmé, ma soprattutto a Valery, come ha mostrato il manoscritto di una lezione tenuta in Svizzera nel 1944. Il presente contributo ricostruisce l'origine di queste idee attraverso lo studio delle correzioni del manoscritto della "Critica degli scartafacci" (l'articolo pubblicato nel 1948 in polemica con Croce), e offre al contempo un esempio illuminante - una vera e propria mise en abyme - di "come lavorava Contini". 